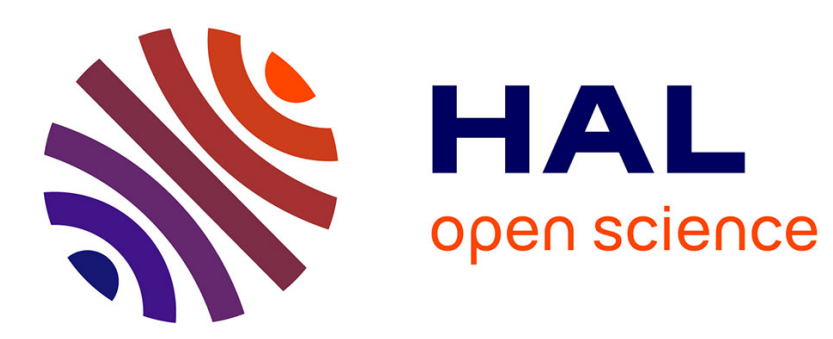

\title{
APPLICATION DE LA NEURO-INFORMATIQUE À L'ANALYSE DES COMPOSANTS DE L'ENVIRONNEMENT SONORE URBAIN
}

\author{
C. Arras, B. Mariusse
}

\section{- To cite this version:}

C. Arras, B. Mariusse. APPLICATION DE LA NEURO-INFORMATIQUE À L'ANALYSE DES COMPOSANTS DE L'ENVIRONNEMENT SONORE URBAIN. Journal de Physique IV Proceedings, 1992, 02 (C1), pp.C1-445-C1-448. 10.1051/jp4:1992195 . jpa-00251267

HAL Id: jpa-00251267 https://hal.science/jpa-00251267

Submitted on 1 Jan 1992

HAL is a multi-disciplinary open access archive for the deposit and dissemination of scientific research documents, whether they are published or not. The documents may come from teaching and research institutions in France or abroad, or from public or private research centers.
L'archive ouverte pluridisciplinaire HAL, est destinée au dépôt et à la diffusion de documents scientifiques de niveau recherche, publiés ou non, émanant des établissements d'enseignement et de recherche français ou étrangers, des laboratoires publics ou privés. 


\title{
APPLICATION DE LA NEURO-INFORMATIQUE À L'ANALYSE DES COMPOSANTS DE L'ENVIRONNEMENT SONORE URBAIN
}

\author{
C. ARRAS et B. MARIUSSE*
}

ACOUPHEN S.A., Ingénierie en Acoustique et Vibrations, Centre d'Entreprise et d'Innovation, BP. 2132, F-69603 Villeurbanne cedex, France

${ }^{*}$ COURBON S.A., Automatique et Informatique de Production, 2 rue Hector Berlioz, BP. 327, F-42105 St Etienne cedex 2, France

Measurements of noise level lead to quantify the community noise potential, but are not sufficient to qualify the related community response. Such response depends on many parameters (social, cultural, urban, physical,...).

Hence, building up a methodology should allow to define, store, sort and analyse all such parameters and their interrelationships, both influencing the community noise response.

A Multiple Neural Network Learning System is used in the development of an adapted methodology and in its applications. The potential of the proposed approach is emphasized through the case study of a part of Lyon city, representative of a typical urban situation. This work is aiming at developing a decision assisting tool in the acoustical design of urban areas.

\section{1- OBJECTIFS DU PROJET}

Gérer et concevoir l'espace sonore urbain ne saurait se réduire à une question d'acoustique physique. La notion de gêne associée à la nuisance sonore a une connotation sociale et culturelle qui la rend fortement dépendante des caractéristiques du milieu dans lequel la nuisance est générée et reçue.

Il est important de mettre en oeuvre une méthodologie qui permette de définir, stocker, trier et analyser l'ensemble des paramètres physiques, urbanistiques et socioculturels, ainsi que leurs interrelations, contribuant à influencer la sensation de gêne sonore perçue par la population.

La neuro-informatique peut apporter des réponses fiables dans des domaines où les approches classiques de traitement de données s'avèrent inefficaces (phénomène complexe non modélisable avec données bruitées).

Ce projet n'a pas la prétention d'identifier de manière exhaustive toutes les caractéristiques d'un espace sonore urbain, ni toutes leurs interrelations, mais plutôt d'étudier la faisabilité d'une méthode d'approche simple, ouverte et évolutive. 


\section{2 - LA NEURO-INFORMATIQUE COMME SUPPORT ET OUTIL DE DEVELOPPEMENT}

\section{2-1 - Généralités}

La Neuro-informatique ou "informatique connexionniste" est une discipline scientifique se situant au carrefour de la Biologie et de l'Informatique. La Neuro-informatique met en oeuvre des réseaux neuromimétiques organisés en couches selon une structure dépendant de l'objectif à atteindre simulant les fonctions du cerveau humain.

La réalisation d'une application se décompose en trois grandes phases : l'apprentissage, la reconnaissance supervisée, le fonctionnement autonome et la mise à jour.

Dans la phase d'apprentissage, on fournit un nombre d'exemples judicieusement choisis, contenant à la fois la cause et l'effet, c'est à dire l'entrée et la sortie correspondante. Le réseau s'organise de manière à obtenir en sortie la réponse souhaitée en fonction des entrées proposées et se constitue une mémoire.

La phase de reconnaissance consiste à tester la validité de l'apprentissage sur un échantillonnage d'exemples non encore appris et à lever les ambiguïtés éventuelles.

Dans la troisième phase, le système peut alors fonctionner de façon autonome et peut toujours être enrichi de situations nouvelles.

\section{2-2- Intérêt}

Cette technique est la première alternative viable au calcul programmé séquentiel utilisé classiquement en Intelligence Artificielle. Elle s'adresse aux problèmes pour lesquels on sait peu de choses sur les algorithmes ou raisonnements à appliquer. Elle s'accommode de données imprécises ou bruitées et s'autoadapte au problème à résoudre. La reconnaissance de formes et la prévision économique sont ses domaines privilégiés. La méthode d'apprentissage qui peut se résumer par la "connaissance par l'exemple" engendre un gain de temps et donc de coût de développement.

\section{2-3 - Le logiciel utilisé}

NESTOR (NEurone STORage) est un logiciel neuro-mimétique dédié à la classification. Il permet de séparer des catégories de figures ayant une frontière hautement imbriquée, sans avoir à se soucier des relations complexes pouvant exister entre caractéristiques.

Dans le cas de la présente étude, NESTOR conduit à évaluer et sélectionner les paramètres pertinents et, d'autre part, à les pondérer et les classer sur une échelle représentative d'un potentiel de gêne sonore. Le caractère incrémental de l'apprentissage et la possibilité de modifier le nombre des paramètres d'entrée et sortie permet un réajustement permanent de la connaissance à priori du domaine étudié.

\section{3 - PRINCIPE ET METHODES DE L'ETUDE}

\section{3-1 - Analyse du problème}

Pour décrire le bruit les acousticiens ont développé des méthodes de mesure, des outils ou modèles de simulation. La gêne réellement ressentie par les individus est une réponse à une situation globale liée à la perception des indicateurs de bruit mais auusi à l'analyse des informations fournies selon sa propre culture et son vécu.

L'environnement sonore urbain est caractérisé par une infinité de sources émettant des sons de nature, d'intensité et de périodicité différentes, qui se propagent dans un milieu physique dont les caractéristiques géographiques, urbanistiques et architecturales déterminent des facteurs de forme, d'orientation, d'absorption, etc... Certaines sources dominantes et identifiables (principalement relatives aux transports et aux activités économiques) peuvent être listées et caractérisées par les paramètres adéquats, mais la plupart des sources sonores ne sont pas identifiables individuellement ; or, elles contribuent largement à la "rumeur de la ville" et participent à l'identité sonore d'un site.

Cette rapide analyse permet de distinguer trois familles de paramètres descripteurs :

- du milieu urbanistique et architectural

- du milieu socioculturel

- des caractèristiques des sources sonores identifiables (nature, intensité, périodicité) 


\section{3-2 - Approche méthodologique}

En tout point de l'espace urbain (plan de zone), on doit pouvoir définir la valeur d'un certain nombre de paramètres descripteurs ayant une incidence sur le milieu sonore, la façon dont il est perçu et donc le potentiel de gêne résultant en ce point.

Dans les enquêtes effectuées par les psychoacousticiens sur des situations particulières (impact d'une voirie urbaine par exemple), on propose un classement quantitatif de la gêne sur une échelle théorique, mais on peut imaginer parallélement un classement qualitatif, la gêne pouvant résulter d'un facteur essentiel et bien repéré , ou être le résultat d'une addition de situations défavorables .

La figure ci-après représente schématiquement le principe de fonctionnement de l'application.

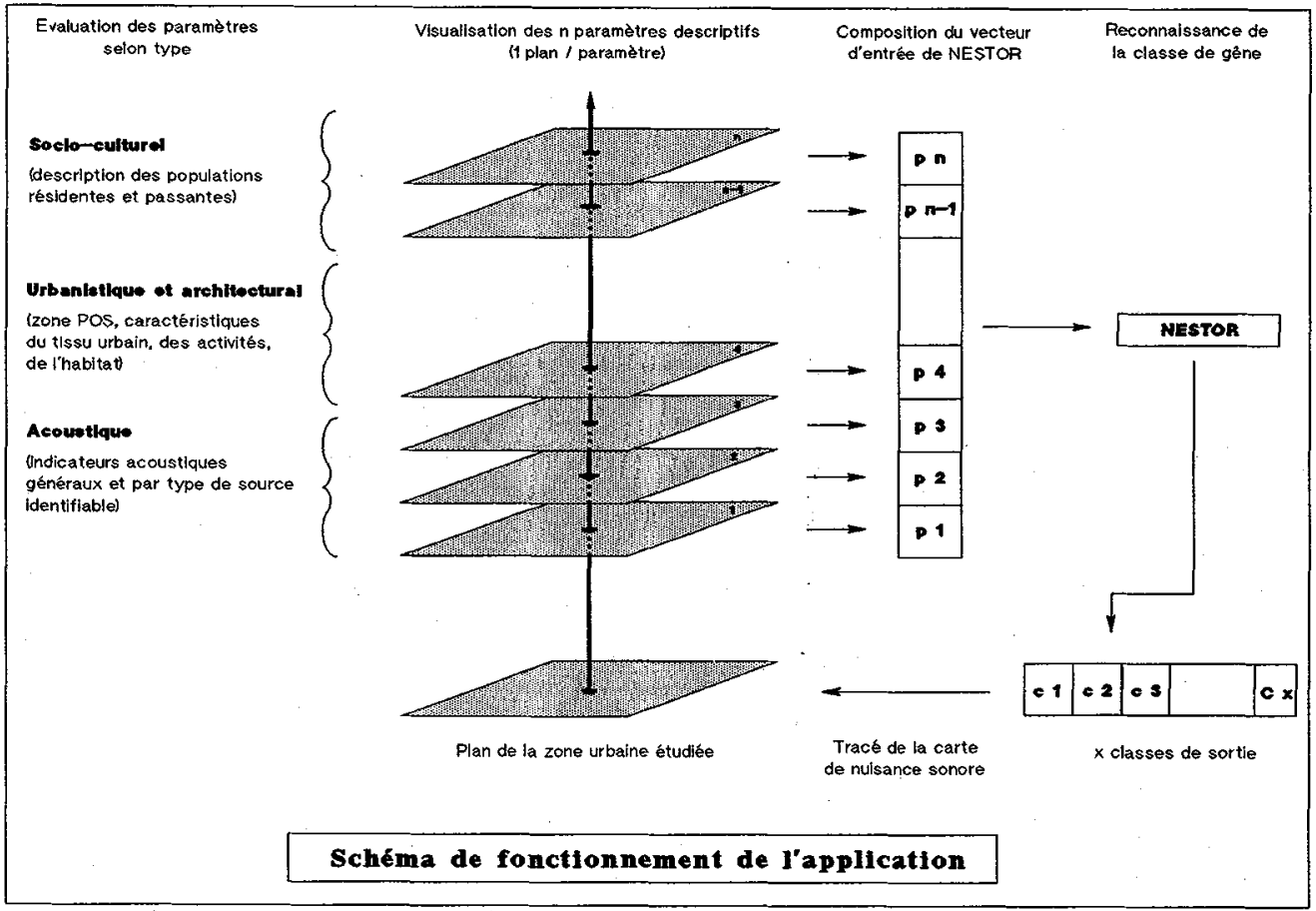

Le système fonctionnant par apprentissage, il s'agit donc de lui fournir un nombre suffisant d'exemples pertinents afin de lui permettre de se constituer une mémoire.

Des étapes successives d'apprentissage et de test sont menées sur des sous-ensembles de paramètres, afin de tester le poids de chacun, de remettre éventuellement en cause certains ou d'en introduire de nouveaux.

Un support cartographique est utilisé pour visualiser les paramètres d'entrée ainsi que les classes de sortie.

\section{4 - APPLICATION ET DEVELOPPEMENT}

\section{4-1 - Choix d'un site pilote}

Le septième arrondissement de la ville de LYON, choisi comme site pilote, offre des composantes très variées et représentatives d'un espace sonore urbain type, ainsi que de nombreux projets d'aménagement et de restructuration.

Sur ce site, un travail de recueil de données est mené : Plan d'Occupation des Sols, études de quartier, données INSEE, mesures de niveaux sonores, inventaires des infrastructures de transport, des activités économiques, fichier des plaintes, etc...

Dans un deuxième temps, il faudra investir d'autres sites afin d'apporter des éléments complémentaires à l'apprentissage pour la validation de la méthode. 


\section{4-2 - Essais préliminaires}

\section{4-2-1 - Simulation à partir de la réglementation}

La notion de gêne n'étant pas mesurable avec un appareil physique, de nombreuses études ont été menées, dans le cadre de l'élaboration de la réglementation, pour tenter de fixer des limites en delà desquelles il y a présomption de nuisance acoustique.

Dans un premier temps, la réglementation en vigueur, applicable pour les installations classées, infrastructures de transport, bruits de voisinage, définit des paramètres, notamment l'indicateur de bruit ambiant et l'indicateur d'émergence. Les échantillons d'apprentissage et de test sont réalisés par simulation de situations, dans lesquelles les classes de gêne sont appréciées selon les valeurs des indicateurs normalisés.

Les premiers essais portant sur des caractéristiques d'ordre plutôt acoustiques montrent que le système est capable de reconnaître les classes sans rien savoir de la relation paramètres/classes, et que la classe où les deux indicateurs "règlementaires" sont dépassés est la moins bien cernée.

\section{4-2-2-Application sur le site pilote}

Les paramètres d'entrée choisis initialement caractérisent le tissu urbain et l'activité qui s'y développe : coefficient d'occupation des sols, homogénéité du tissu urbain, hauteur moyenne des bâtiments, ancienneté de la construction, activités de la zone, présence d'équipements collectifs..

Le travail consiste à lier ces paramètres avec ceux utilisés précédemment en s'appuyant sur des mesures de niveau sonore, le fichier des plaintes, des calculs prévisionnels en matière de bruits routier et ferroviaire, etc... et à développer la visualisation cartographique des paramètres (voir figure).

L'intégration de la dimension socioculturelle du problème, la plus délicate à prendre en compte, devra se faire en s'appuyant par exemple sur des résultats d'enquêtes.

\section{5 - CONCLUSIONS}

La complexité du travail réside dans la difficulté d'appréciation de la nuisance sonore, donc dans la difficulté d'élaborer des exemples d'apprentissage pertinents.

Les directions de recherche étant multiples, le problème est décomposé en petites études, intégrées peu à peu à l'ensemble.

La méthode proposée s'accommode bien du flou des données, elle permet une approche progressive du problème et assure le suivi de son évolution dans le temps et dans l'espace. A partir des connaissances acquises et mémorisées, le système peut poursuivre son apprentissage sur d'autres sites et enrichir sa mémoire.

A terme, un tel outil conduira à l'élaboration d'un système d'aide à la conception acoustique de sites urbains.

Ce projet est partiellement financé par le Ministère de l'Environnement français. 